\title{
EFFECT OF SOME SUGARS AND AGRO-INDUSTRIAL BY- PRODUCTS ON CAROTENOIDS PRODUCTION BY SOME YEAST STRAINS OF RHODOTORULA SPP.
}

Selim,A. E. I.; M. M. Kassem; Samia M. M. Bayoumy; M. M. A. EISawah and Dina F. I. Ali

Dept. of Agric. Microbiol., Fac. of Agric., Mansoura Univ., Mansoura , Egypt.

\begin{abstract}
The production of carotenoids by Rhodotorula glutinis NRRL Y-842, $R$. armeniaca NRRL Y-17201, $R$. mucilaginosa NRRL Y-844, $R$. bogoriensis NRRL Y5980 or $R$. aurantiaca NRRL Y-1584 on glucose as a carbon source were investigated at different incubation periods at $30^{\circ} \mathrm{C}$ in shaking incubator. Among the different yeast strains, R.glutinis NRRL Y-842 proved to be the most active producer during the fermentation period, the maximum values of carotenoids produced by Rhodotorula strains were $2328.29 \mu \mathrm{g} / \mathrm{L}$ at $96 \mathrm{hr} ., 10.99 \mu \mathrm{g} / \mathrm{L}$ at $96 \mathrm{hr} ., 10.99 \mu \mathrm{g} / \mathrm{L}$ at $96 \mathrm{hr} ., 71.13 \mu \mathrm{g} / \mathrm{L}$ at $120 \mathrm{hr}$. and $62.73 \mu \mathrm{g} / \mathrm{L}$ at $96 \mathrm{hr}$., respectively. Using sucrose, lactose, maltose or fructose as a carbon source, $R$. glutinis NRRL Y-842 produced $732.45 \mu \mathrm{g} / \mathrm{L}$ at $72 \mathrm{hr}$., $488.88 \mu \mathrm{g} / \mathrm{L}$ at $72 \mathrm{hr} ., 1704.97 \mu \mathrm{g} / \mathrm{L}$ at $96 \mathrm{hr}$. and $2166.01 \mu \mathrm{g} / \mathrm{L}$ at $96 \mathrm{hr}$. , respectively. When agro- industrial by-products, namely, untreated sugarcane molasses, untreated beet molasses, treated sugarcane molasses, treated beet molasses, untreated vinasse, treated vinasse, glucose syrup, soy bean flour extract (SFE) and maize flour extract (MFE) were also used as a carbon source, the maximum values of carotenoids produced by $R$. glutinis NRRL Y-842 were $1772.19 \mu \mathrm{g} / \mathrm{L}$ at $120 \mathrm{hr} ., 1571.40 \mu \mathrm{g} / \mathrm{L}$ at $144 \mathrm{hr} ., 1002.01 \mu \mathrm{g} / \mathrm{L}$ at $120 \mathrm{hr} ., 679.00 \mu \mathrm{g} / \mathrm{L}$ at $96 \mathrm{hr} ., 916.65 \mu \mathrm{g} / \mathrm{L}$ at $120 \mathrm{hr}$. $452.89 \mu \mathrm{g} / \mathrm{L}$ at $120 \mathrm{hr} ., 1831.36 \mu \mathrm{g} / \mathrm{L}$ at $96 \mathrm{hr} ., 426.80 \mu \mathrm{g} / \mathrm{L}$ at $48 \mathrm{hr}$. and $340.79 \mu \mathrm{g} / \mathrm{L}$ at $48 \mathrm{hr}$., respectively.

Keywords: Carotenoids, Rhodotorula spp., Molasses, vinasse.
\end{abstract}

\section{INTRODUCTION}

Carotenoids are a group of natural pigments produced by a wide range of microorganisms and plants. They are used commercially as food colorants and for their pharmaceutical and chemical role, not only they act as vitamin A precursors, but also for their coloring, antioxidant, possible tumor- inhibiting activity, involvement in the visual attraction of animals such as flower pollinators or mating partners(Johnson and Schroeder,1995;Ferrao and Garg,2011). Many carotenoids are being produced by chemical synthesis, which yields products that are pure and cheap (Gordon and Pauerfeind, 1982).

The international market of carotenoids has been met mainly by synthetic carotenoids with structures identical to those of natural carotenoids, but there is growing demand for natural sources. Microbial carotenoids have been studied and their potentials recognized over the years. Several microorganisms, including bacteria, algae, moulds and yeasts of the genera Rhodotorula, Rhodosporidium, Sporobolomyces and Phaffia are able to produce carotenoids naturally( Frengova and Beshkova, 2009). The 
Selim,A. E. I. et al.

synthesis of different natural important carotenoids by several yeasts species belonging to the genera Rhodotorula and Phaffia, has led to consider these microorganisms as a potential pigment sources (Catalina Voaides and Dima, 2012).

The commercial utilization of microorganisms with bio-technological potential to produce carotenoids is presently limited by the high cost production. However, the cost of carotenoid production can be minimized by the use of inexpensive industrial by-products as nutrient sources (Aksu and Eren, 2005)

The aim of this study was to examine the potential of some sugars and some agro-industrial by-products as substrates for the production of carotenoids by some yeast strains belonging to the genus Rhodotorula.

\section{MATERIALS AND METHODS}

\section{Yeast strains}

Five yeast strains, namely, Rhodotorula glutinis NRRL Y-842, Rhodotorula armeniaca NRRL Y-17201, Rhodotorula mucilaginosa NRRL Y844, Rhodotorula bogoriensis NRRL Y-5980 and Rhodotorula aurantiaca NRRL Y-1584 were kindly obtained from Cairo Mircen, CAIM, Faculty of Agriculture, Ain- Shams Univ., Cairo, Egypt. These strains were subcultured on YM (ATCC medium 200) slants (dextrose $10.0 \mathrm{~g}$, peptone $5.0 \mathrm{~g}$, yeast extract $3.0 \mathrm{~g}$, malt extract $3.0 \mathrm{~g}$, agar $15.0 \mathrm{~g}$, distilled water $1.0 \mathrm{~L}$ ) at $28^{\circ} \mathrm{C}$, maintained at $4^{\circ} \mathrm{C}$ and subcultured every 3 weeks.

\section{Production medium}

25.0 g glucose, 10.0 g yeast extract, $2.0 \mathrm{~g} \mathrm{~K}_{2} \mathrm{HPO}_{4}, 2.0 \mathrm{~g} \mathrm{KH}_{2} \mathrm{PO}_{4}, 0.1$ $\mathrm{g} \mathrm{MgSO}_{4} .7 \mathrm{H} 2 \mathrm{O}, 1.0 \mathrm{~L}$ distilled water. The $\mathrm{pH}$ of the medium was adjusted at 6 (Bhosale and Gadre, 2001a). The medium was sterilized at $100 \stackrel{\circ}{\circ}$ for 20 min. 3 times with $24 \mathrm{hr}$. interval. Furthermore $400 \mu \mathrm{g} / \mathrm{L}$ of thiamine and 23 $\mu \mathrm{g} / \mathrm{L}$ of $\mathrm{P}$-aminobenzoic acid were added to the production medium in case of all strains except Rhodotorula glutinis NRRL Y-842 (Peterson et al., 1958).

\section{Preparation of standard inoculum}

Inocula were obtained from cultures grown on $\mathrm{YM}$ slants at $28^{\circ} \mathrm{C}$ for $48 \mathrm{hr}$. A loop of the yeast cells was transferred to $5 \mathrm{ml}$ of production medium and incubated at $28^{\circ} \mathrm{C}$ for $24 \mathrm{hr}$., transferred to $100 \mathrm{ml}$ of fermentation medium in $250 \mathrm{ml}$ Erlenmeyer flask. Inoculated flasks were incubated in a rotary shaking incubator (Lab-Line Incubator-Shaker) at $30^{\circ} \mathrm{C} / 150 \mathrm{rpm}$. Samples were taken every $24 \mathrm{hr}$. interval through 6 days, and the following parameters of fermentation were studied.

\section{III.1. Time course}

To investigate carotenoids production by Rhodotorula strains, the cultures of the used five strains cultivated on fermentation medium were incubated for 6 days at $30^{\circ} \mathrm{C}$. The cell dry weight, carotenoids, $\mathrm{pH}$ and consumed sugars were investigated daily for each strain. 


\section{III.2. Carbon source \\ III.2.1. Sugars}

To investigate the effect of some sugars on carotenoids production by Rhodotorula glutinis NRRL Y-842, glucose was replaced by sucrose, lactose, maltose or fructose. Each source was added to the fermentation medium equivalent to $25 \mathrm{gm}$ glucose /L. The final volume of each fermentation medium was adjusted at $\mathrm{pH} 6$, distributed in flasks, sterilized at $100^{\circ} \mathrm{C}$ for 20 min. 3 times with $24 \mathrm{hr}$. interval, inoculated and incubated at $30^{\circ} \mathrm{C}$ for 6 days.

\section{III.2.2. Molasses}

According to the method of Bhosale and Gadre (2001b), sugarcane and beet molasses were used for fermentation experiments. They were diluted 2 folds (W/V) using distilled water. Then each was divided into 2 parts, the $\mathrm{pH}$ of the first part was adjusted to 2.0 with $5 \mathrm{~N} \mathrm{HCl}$ and it was held in boiling water bath for $40 \mathrm{~min}$. for sucrose hydrolysis. After hydrolysis, the solution was cooled to room temperature and its $\mathrm{pH}$ was adjusted to 6.0 with $1 \mathrm{~N} \mathrm{NaOH}$, The precipitate was removed by centrifugation. Total reducing sugar (TRS) was assayed spectrophoto-metrically using Somogyi (1952) method in both treated and untreated molasses. After addition of $2 \mathrm{~g} / \mathrm{L}$ of both $\mathrm{K}_{2} \mathrm{HPO}_{4}$ and $\mathrm{KH}_{2} \mathrm{PO}_{4}$, the solutions were sterilized at $100^{\circ} \mathrm{C}$ for 20 min. 3 times with $24 \mathrm{hr}$. interval and used as a fermentation medium.

\section{III.2.3. Glucose syrup}

When glucose syrup was used for fermentation experiments, it was diluted 2 folds (W/V) using distilled water, TRS were measured, and then they were added to the fermentation medium instead of glucose in equivalent concentration. The medium was sterilized at $100^{\circ} \mathrm{C}$ for $20 \mathrm{~min}$. 3 times with 24hr. interval.

\section{III.2.4. Soy bean flour and maize flour}

According to Buzzini and Martini(1999), $1.5 \mathrm{~L}$. Sulfuric acid (0.2 N) was added to $250 \mathrm{~g}$ of each of soy bean flour and maize flour, then autoclaved on $121 . \mathrm{C}$ for $15 \mathrm{~min}$., cooled and centrifuged at $5000 \mathrm{rpm}$ for 10 minutes. In case of MFE, any carotenoid traces were extracted using di-ethyl ether in order to remove possible traces of carotenoids, then centrifuged and added to the fermentation medium without glucose to reach a TC concentration of $25 \mathrm{~g} / \mathrm{L}$. The medium was sterilized at $100^{\circ} \mathrm{C}$ for $20 \mathrm{~min}$. 3 times with $24 \mathrm{hr}$. interval.

\section{Extraction of carotenoids}

According to the method of Park et al. (2005), modified by Ali (2013), to extract the carotenoids, $50 \mathrm{ml}$ of each fermented liquor was centrifuged at $3500 \mathrm{rpm}$ for $15 \mathrm{~min}$., after decanting the supernatant, the precipitate was washed twice by distilled water and the washing-water was discarded after centrifugation every time. The precipitated cells were broken up with a glass rod after adding $10 \mathrm{ml}$ of $0.5 \mathrm{~N} \mathrm{HCl}$, boiled in water bath for $15 \mathrm{~min}$., cooled in ice water for 10 minutes. For extraction of the pigments, measured amounts of acetone were added and the cells stirred vigorously. To ensure complete extraction of the pigments, more amounts of acetone were added until cells 
turn colorless. Amounts of di-ethyl ether equal to those of acetone were added to the previous, and moved to a separation funnel. Then amounts of cold $\mathrm{NaCl}$ solution (15\%) were added for purification of di-ethyl ether layer, and then the di-ethyl ether layer was taken and assayed spectrophotometrically at $455 \mathrm{~nm}$. (JENWAY 6305 UV/vis. Spectrophotometer). The standard curve was blotted using $\beta$-carotene as a standard substrate. Amounts of carotenoids were calculated using standard curve.

\section{Fermentation aspects}

At certain time intervals $(24,48,72,96,120$ and $144 \mathrm{hr}$.), the following fermentation aspects were done.

\section{V.1. Cell dry weight}

The cell dry weight was determined in $50 \mathrm{ml}$ of the fermented culture by centrifugation at $3500 \mathrm{rpm}$ for $15 \mathrm{~min}$., washing the sediment twice with distilled water and dried at $85 \stackrel{\circ}{\circ}$ to constant weight.

\section{V.2. Residual sugars}

Residual sugars were determined in the centrifuged fermented liquor after removing cells, according to the method of Somogyi (1952) using glucose as a standard.

V.3. $\mathrm{pH}$ meter.

The initial and final $\mathrm{pH}$ was measured with JENWAY 3505 digital $\mathrm{pH}$

N.B: all experiments were repeated three times, and the reported data were averages.

\section{RESULTS AND DISCUSSION}

Total carotenoids produced by the five Rhodotorula strains, using glucose as a sole carbon source were listed in Table 1. Results show that Rhodotorula glutinis NRRL Y-842 yielded the highest value $(2328.29 \mu \mathrm{g} / \mathrm{L})$ at $96 \mathrm{hr}$. On the other hand, the highest values obtained by Rhodotorula armeniaca NRRL Y-17201, Rhodotorula mucilaginosa NRRL Y-844, Rhodotorula bogoriensis NRRL Y-59880 and Rhodotorula aurantiaca NRRL Y-1984 were 10.99,10.99,71.13 and $62.73 \mu \mathrm{g} / \mathrm{L}$ at 96hr., 96hr., 120hr. and $96 \mathrm{hr} .$, respectively. Also, data show that the highest values of consumed sugars $(\mathrm{gm} / \mathrm{Liter})$ and carotenoids produced $(\mu \mathrm{g})$ per gm consumed sugar were obtained by Rhodotorula glutinis NRRL Y-842, being $24.734 \mathrm{~g} / \mathrm{L}$ and $94.133 \mu \mathrm{g} / \mathrm{g}$, respectively. It could be stated that, Rhodotorula glutinis NRRL $\mathrm{Y}-842$ proved to be the best strain for carotenoids production. So, other sugars were used to study their effect on carotenoids production by this yeast strain.

Data in Table 2 show the effect of some sugars used as a carbon source, namely, sucrose, lactose, maltose and fructose on carotenoids production by Rhodotorula glutinis NRRL Y-842. Among the used sugars, fructose recorded the highest value of carotenoids $2166.01 \mu \mathrm{g} / \mathrm{L}$ at $96 \mathrm{hr}$., while sucrose, lactose and maltose recorded $732.45,488.88$ and 1704.97 $\mu \mathrm{g} / \mathrm{L}$ at $72 \mathrm{hr} ., 72 \mathrm{hr}$. and $96 \mathrm{hr}$., respectively. Also, by using fructose as a 
carbon source, Rhodotorula glutinis NRRL Y-842 recorded the highest level of cell dry weight $(11.712 \mathrm{~g} / \mathrm{L})$ and carotene / cell dry weight $(184.94 \mu \mathrm{g} / \mathrm{gm})$.

Data in Tables 3a\&b show the effect of using some agro-industrial byproducts on carotenoids production by Rhodotorula glutinis NRRL Y-842. Data reveal that the highest values of carotenoids were recorded with using untreated sugarcane molasses $(1772.19 \mu \mathrm{g} / \mathrm{L}$ at $120 \mathrm{hr}$.), untreated beet molasses (1571.4 $\mu \mathrm{g} / \mathrm{L}$ at $144 \mathrm{hr}$.) and treated sugarcane molasses $(1002.01 \mu \mathrm{g} / \mathrm{L}$. at $120 \mathrm{hr}$.). $\beta$-Carotene accumulation in yeast increased when mineral-rich molasses was supplied (Bhosale and Gadre, 2001a).

Table 1: Production of total carotenoids by yeast strains of Rhodotorula spp. grown on media containing glucose as a carbon source

\begin{tabular}{|c|c|c|c|c|c|c|c|}
\hline Strain & Time & $\begin{array}{c}\text { Cell dry } \\
\text { weight } \\
\text { (gm/liter) }\end{array}$ & $\begin{array}{c}\text { Carotenoids } \\
\text { ( } \mu \mathrm{g} / \mathrm{liter})\end{array}$ & $\begin{array}{c}\text { Carotene/cell } \\
\text { dry weight } \\
(\mu \mathrm{g} / \mathrm{gm})\end{array}$ & $\begin{array}{c}\text { Final } \\
\mathrm{pH}\end{array}$ & $\begin{array}{c}\text { Consumed } \\
\text { sugars } \\
\text { (gm/liter) }\end{array}$ & $\begin{array}{c}\text { Carotene / } \\
\text { Consumed } \\
\text { Sugar } \\
(\mu \mathrm{g} / \mathrm{gm})\end{array}$ \\
\hline \multirow{4}{*}{$\begin{array}{l}\text { Rhodotorula } \\
\text { glutinis }\end{array}$} & $24 \mathrm{hr}$. & 00.6600 & 0050.44 & 076.42 & 5.92 & 12.121 & 004.161 \\
\hline & $48 \mathrm{hr}$. & 07.1880 & 0757.47 & 105.38 & 5.89 & 24.071 & 031.468 \\
\hline & $72 \mathrm{hr}$. & 09.7120 & 1358.39 & 139.87 & 5.76 & 24.693 & 055.011 \\
\hline & $96 \mathrm{hr}$. & 11.9620 & 2328.29 & 194.64 & 6.21 & 24.734 & 094.133 \\
\hline \multirow[t]{2}{*}{ NRRL Y-842 } & $120 \mathrm{hr}$. & 08.2900 & 1038.87 & 125.32 & 6.40 & 24.782 & 041.920 \\
\hline & $144 \mathrm{hr}$. & 04.7160 & 0642.92 & 136.33 & 7.60 & 24.791 & 025.933 \\
\hline \multirow{4}{*}{$\begin{array}{l}\text { Rhodotorula } \\
\text { armeniaca }\end{array}$} & $24 \mathrm{hr}$. & 00.0400 & 06.47 & 161.75 & 5.88 & 01.038 & 006.233 \\
\hline & $48 \mathrm{hr}$. & 00.2000 & 06.47 & 032.35 & 5.80 & 01.447 & 004.471 \\
\hline & $72 \mathrm{hr}$. & 00.2200 & 09.70 & 044.09 & 5.78 & 01.666 & 005.822 \\
\hline & 96hr. & 00.9800 & 10.99 & 011.21 & \begin{tabular}{|l|}
5.67 \\
\end{tabular} & 03.176 & 003.460 \\
\hline \multirow[t]{2}{*}{ NRRL Y-17201 } & $120 \mathrm{hr}$. & 00.1000 & 02.26 & 022.60 & 5.85 & 03.396 & 000.665 \\
\hline & $144 \mathrm{hr}$. & 00.0600 & 02.26 & 037.67 & 5.75 & 03.491 & 000.647 \\
\hline \multirow{4}{*}{$\begin{array}{l}\text { Rhodotorula } \\
\text { mucilaginosa }\end{array}$} & $24 \mathrm{hr}$. & 00.4333 & 06.47 & 014.93 & 5.89 & 03.239 & 001.998 \\
\hline & $48 \mathrm{hr}$. & 00.8467 & 06.47 & 007.64 & 5.82 & 03.522 & 001.837 \\
\hline & $72 \mathrm{hr}$. & 04.8067 & 09.70 & 002.02 & \begin{tabular}{|l|}
5.74 \\
\end{tabular} & 04.182 & 002.319 \\
\hline & $96 \mathrm{hr}$. & 07.1333 & 10.99 & 001.54 & 6.08 & 04.434 & 002.479 \\
\hline \multirow[t]{2}{*}{ NRRL Y-844 } & 120hr. & 03.9467 & 02.26 & 000.57 & 5.84 & 04.654 & 000.486 \\
\hline & 144hr. & 01.6400 & 02.26 & 001.38 & 6.02 & 05.629 & 000.401 \\
\hline \multirow{4}{*}{$\begin{array}{l}\text { Rhodotorula } \\
\text { bogoriensis }\end{array}$} & $24 \mathrm{hr}$. & 00.0133 & 04.20 & 315.79 & 5.82 & 00.943 & 004.454 \\
\hline & $48 \mathrm{hr}$. & 00.8600 & 06.47 & 007.52 & 6.50 & 03.176 & 002.037 \\
\hline & $72 \mathrm{hr}$. & 01.4533 & 15.20 & 010.46 & \begin{tabular}{|l|}
6.67 \\
\end{tabular} & 03.585 & 004.240 \\
\hline & $96 \mathrm{hr}$. & 01.8133 & 34.60 & 019.08 & 6.96 & 03.742 & 009.246 \\
\hline \multirow[t]{2}{*}{ NRRL Y-5980 } & $120 \mathrm{hr}$. & 07.5333 & 71.13 & 009.44 & 5.08 & 08.648 & 008.225 \\
\hline & 144hr. & 05.2800 & 29.10 & 005.51 & 5.14 & 09.591 & 003.034 \\
\hline \multirow{4}{*}{$\begin{array}{l}\text { Rhodotorula } \\
\text { aurantiaca }\end{array}$} & $24 \mathrm{hr}$. & 00.6000 & 02.26 & 003.77 & 4.78 & 00.880 & 002.568 \\
\hline & $48 \mathrm{hr}$. & 00.7000 & 06.47 & 009.24 & 4.28 & 01.038 & 006.233 \\
\hline & $72 \mathrm{hr}$. & 00.7267 & 09.70 & 013.35 & 4.22 & 01.289 & 007.525 \\
\hline & 96hr. & 00.8667 & 62.73 & 072.38 & 4.20 & 02.138 & 029.340 \\
\hline \multirow[t]{2}{*}{ NRRL Y-1584 } & $120 \mathrm{hr}$. & 00.5933 & 19.40 & 032.70 & 4.26 & 02.767 & 007.011 \\
\hline & $144 \mathrm{hr}$. & 00.4267 & 04.20 & 009.84 & 4.22 & 03.491 & 001.203 \\
\hline
\end{tabular}

initial $\mathrm{pH}=6$, at 30 C

Thus cheap, available nutrient sources such as sugarcane and beet molasses can be used to make the process of pigment production industrially 
Selim,A. E. l. et al.

feasible. The highest production recorded by using glucose syrup $(1831.36 \mu \mathrm{g} / \mathrm{L})$ may be attributed to the nature of glucose syrup. Glucose syrup has unique sugar content, that it contains monosaccharides, disaccharides, tetrasaccharides, pentasaccharides, hexasaccharides and high sugars (Johnson and Schroeder, 1995).

Table 2: Effect of some sugars on carotenoids production by Rhodotorula glutinis NRRL Y-842

\begin{tabular}{|c|c|c|c|c|c|c|c|}
\hline $\begin{array}{l}\text { Carbon } \\
\text { source }\end{array}$ & Time & $\begin{array}{c}\text { Cell dry } \\
\text { weight } \\
\text { (gm/litre) }\end{array}$ & $\begin{array}{c}\text { Carotenoids } \\
(\mu \mathrm{g} / \mathrm{litre})\end{array}$ & $\begin{array}{l}\text { Carotene/cell } \\
\text { dry weight } \\
(\mu \mathrm{g} / \mathrm{gm})\end{array}$ & $\begin{array}{c}\text { Final } \\
\text { pH }\end{array}$ & $\begin{array}{l}\text { Consumed } \\
\text { sugars } \\
\text { (gm/liter) }\end{array}$ & $\begin{array}{c}\text { Carotene / } \\
\text { Consumed } \\
\text { Sugar } \\
(\mu \mathrm{g} / \mathrm{gm}) \\
\end{array}$ \\
\hline \multirow{6}{*}{ Sucrose } & $24 \mathrm{hr}$. & 01.6580 & 0164.90 & 099.46 & 6.36 & 22.825 & 007.225 \\
\hline & $48 \mathrm{hr}$. & 02.3440 & 0224.94 & 095.96 & 8.07 & 23.039 & 009.763 \\
\hline & $72 \mathrm{hr}$. & 05.8000 & 0732.45 & 126.29 & 6.55 & 23.359 & 031.356 \\
\hline & $96 \mathrm{hr}$. & 03.4040 & 0501.39 & 147.29 & 7.95 & 23.611 & 021.235 \\
\hline & $120 \mathrm{hr}$. & 02.2572 & 0253.27 & 112.21 & 6.14 & 24.000 & 010.553 \\
\hline & 144hr. & 01.5360 & 0031.62 & 020.59 & 8.44 & 24.175 & 001.308 \\
\hline \multirow{6}{*}{ Lactose } & $24 \mathrm{hr}$. & 01.2680 & 0140.26 & 110.62 & 6.16 & 02.699 & 051.967 \\
\hline & $48 \mathrm{hr}$. & 02.3600 & 0420.40 & 178.14 & 7.84 & 05.619 & 074.818 \\
\hline & 72hr. & 03.3740 & 0488.88 & 144.90 & 8.04 & 05.885 & 083.072 \\
\hline & $96 \mathrm{hr}$. & 02.9020 & 0309.33 & 106.60 & 7.91 & 06.328 & 048.883 \\
\hline & $120 \mathrm{hr}$. & 02.6880 & 0164.90 & 061.35 & 8.08 & 07.390 & 022.314 \\
\hline & $144 \mathrm{hr}$. & 02.4140 & 0053.16 & 022.02 & 4.62 & 07.655 & 006.944 \\
\hline \multirow{6}{*}{ Maltose } & $24 \mathrm{hr}$. & 01.4020 & 0145.50 & 103.78 & 6.18 & 16.667 & 008.730 \\
\hline & $48 \mathrm{hr}$. & 04.0120 & 0421.08 & 104.96 & 6.91 & 20.345 & 020.697 \\
\hline & $72 \mathrm{hr}$. & 07.9900 & 0520.02 & 065.08 & 6.28 & 23.776 & 021.871 \\
\hline & $96 \mathrm{hr}$. & 10.0600 & 1704.97 & 169.48 & 6.22 & 24.830 & 068.666 \\
\hline & $120 \mathrm{hr}$. & 07.3780 & 0375.49 & 050.89 & 6.22 & 24.851 & 015.110 \\
\hline & 144hr. & 03.0960 & 0332.32 & 107.34 & 7.35 & 24.894 & 013.349 \\
\hline \multirow{6}{*}{ Fructose } & $24 \mathrm{hr}$. & 01.2480 & 0084.39 & 067.62 & 5.78 & 10.806 & 007.810 \\
\hline & $48 \mathrm{hr}$. & 06.8540 & 0520.89 & 076.00 & 4.70 & 18.472 & 028.200 \\
\hline & $72 \mathrm{hr}$. & 10.2120 & 1385.16 & 135.64 & 5.24 & 23.953 & 057.828 \\
\hline & $96 \mathrm{hr}$. & 11.7120 & 2166.01 & 184.94 & 5.98 & 24.670 & 087.799 \\
\hline & $120 \mathrm{hr}$. & 11.4120 & 1575.28 & 138.04 & 5.96 & 24.682 & 063.823 \\
\hline & 144hr. & 10.6280 & 0774.06 & 072.83 & 6.34 & 24.801 & 031.211 \\
\hline
\end{tabular}

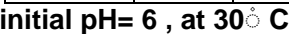

On the other hand the lowest values were noticed with treated vinasse, soy bean flour extract and maize flour extract (452.898, 426.8, $340.79 \mathrm{\mu g} / \mathrm{L}$ at 120, 48 and $48 \mathrm{hr}$.), respectively. Buzzini and Martini (1999) obtained 4.74 and $3.82 \mathrm{mg} / \mathrm{L}$ carotenoids, at $120 \mathrm{hr}$., from R. glutinis DBVPG 6439 cultivated on a medium containing SFE and MFE, respectively. As can be seen, untreated molasses and vinasse promoted the highest amounts of carotenoids while treated molasses and vinasse recorded small amounts of carotenoids in comparable with untreated molasses and vinasse. It can be assumed that metal salts in untreated molasses have a stimulatory effect on carotenoids production and this positive effect is due to a stimulatory effect of cations on carotenoid- synthesizing system. Similar findings were reported by Bhosale and Gadre (2001a). 
Table 3a: Production of carotenoids by Rhodotorula glutinis NRRL Y842 using some agro-industrial raw materials

\begin{tabular}{|c|c|c|c|c|c|c|c|}
\hline $\begin{array}{l}\text { Carbon } \\
\text { source }\end{array}$ & Time & $\begin{array}{c}\text { Cell dry } \\
\text { weight } \\
\text { (gm/litre) }\end{array}$ & $\begin{array}{c}\text { Carotenoids } \\
(\mu \mathrm{g} / \text { litre })\end{array}$ & $\begin{array}{c}\text { Carotene/cell } \\
\text { dry weight } \\
(\mu \mathrm{g} / \mathrm{gm})\end{array}$ & $\begin{array}{c}\text { Final } \\
\mathrm{pH}\end{array}$ & $\begin{array}{c}\text { Consumed } \\
\text { sugars } \\
\text { (gm/liter) }\end{array}$ & $\begin{array}{c}\text { Carotene / } \\
\text { Consumed } \\
\text { Sugar } \\
(\mu \mathrm{g} / \mathrm{gm})\end{array}$ \\
\hline \multirow{6}{*}{$\begin{array}{l}\text { Beet } \\
\text { molasses } \\
\text { (Treated) }\end{array}$} & $24 \mathrm{hr}$. & 1.204 & 0066.93 & 055.59 & 5.48 & 05.675 & 011.794 \\
\hline & $48 \mathrm{hr}$. & 2.176 & 0267.72 & 123.03 & 5.52 & 05.889 & 045.461 \\
\hline & $72 \mathrm{hr}$. & 2.540 & 0446.20 & 175.67 & 5.57 & 06.156 & 072.482 \\
\hline & $96 \mathrm{hr}$. & 2.708 & 0679.00 & 250.74 & 5.71 & 09.529 & 071.256 \\
\hline & $120 \mathrm{hr}$. & 2.304 & 0576.18 & 250.08 & 4.84 & 10.225 & 056.350 \\
\hline & 144hr. & 1.172 & 0261.90 & 223.46 & 5.70 & 11.724 & 022.339 \\
\hline \multirow{6}{*}{$\begin{array}{l}\text { Beet } \\
\text { molasses } \\
\text { (Untreated) }\end{array}$} & 24hr. & 1.824 & 0037.83 & 020.74 & 5.22 & 00.236 & 160.297 \\
\hline & $48 \mathrm{hr}$. & 3.666 & 0439.41 & 119.86 & 5.59 & 02.305 & 190.633 \\
\hline & $72 \mathrm{hr}$. & 4.558 & 0962.24 & 211.11 & 5.59 & 04.728 & 203.520 \\
\hline & 96hr. & 4.782 & 0993.28 & 207.71 & 5.73 & 05.851 & 169.762 \\
\hline & $120 \mathrm{hr}$. & 7.218 & 1067.00 & 147.82 & 5.43 & 14.835 & 071.925 \\
\hline & $144 \mathrm{hr}$. & 16.726 & 1571.40 & 093.95 & 4.60 & 18.558 & 084.675 \\
\hline \multirow{6}{*}{$\begin{array}{l}\text { Sugar cane } \\
\text { molasses } \\
\text { (Treated) }\end{array}$} & 24hr. & 1.694 & 0061.983 & 036.59 & 5.61 & 09.798 & 006.326 \\
\hline & $48 \mathrm{hr}$. & 2.414 & 0214.855 & 089.00 & 5.53 & 15.994 & 013.433 \\
\hline & $72 \mathrm{hr}$. & 2.580 & 0239.590 & 092.86 & \begin{tabular}{|l|}
5.81 \\
\end{tabular} & 18.227 & 013.145 \\
\hline & 96hr. & 3.502 & 0543.200 & 155.11 & 5.78 & 18.444 & 029.451 \\
\hline & $120 \mathrm{hr}$. & 4.192 & 1002.010 & 239.03 & 5.63 & 22.190 & 045.156 \\
\hline & $144 \mathrm{hr}$. & 2.322 & 0649.900 & 279.89 & 5.68 & 22.334 & 029.099 \\
\hline \multirow{6}{*}{$\begin{array}{l}\text { Sugar cane } \\
\text { molasses } \\
\text { (Untreated) }\end{array}$} & 24hr. & 2.082 & 0083.517 & 040.11 & 5.07 & 05.075 & 016.457 \\
\hline & $48 \mathrm{hr}$. & 4.702 & 0491.790 & 104.59 & 5.05 & 15.288 & 032.168 \\
\hline & $72 \mathrm{hr}$. & 5.252 & 0595.483 & 113.38 & 5.88 & 17.293 & 034.435 \\
\hline & $96 \mathrm{hr}$. & 6.750 & 1681.980 & 249.18 & \begin{tabular}{|l|}
5.57 \\
\end{tabular} & 19.048 & 088.302 \\
\hline & $120 \mathrm{hr}$. & 7.316 & 1772.190 & 242.23 & 5.26 & 20.928 & 084.680 \\
\hline & $144 \mathrm{hr}$. & 6.672 & 1488.465 & 223.09 & 5.02 & 21.679 & 068.659 \\
\hline \multirow{6}{*}{$\begin{array}{l}\text { Vinasse } \\
\text { (Treated) }\end{array}$} & $24 \mathrm{hr}$. & 2.758 & 0071.295 & 025.85 & 5.14 & 01.196 & 059.611 \\
\hline & $48 \mathrm{hr}$. & 2.968 & 0115.430 & 038.89 & 4.94 & 01.994 & 057.889 \\
\hline & $72 \mathrm{hr}$. & 3.176 & 0160.050 & 050.39 & 4.94 & 03.657 & 043.765 \\
\hline & $96 \mathrm{hr}$. & 3.422 & 0373.450 & 109.13 & 4.95 & 03.790 & 098.536 \\
\hline & $120 \mathrm{hr}$. & 3.620 & 0452.893 & 125.11 & 4.94 & 04.388 & 103.212 \\
\hline & 144hr. & 2.726 & 0333.195 & 122.23 & 4.94 & 06.250 & 053.311 \\
\hline \multirow{6}{*}{$\begin{array}{l}\text { Vinasse } \\
\text { (Untreated) }\end{array}$} & 24hr. & 2.994 & 0105.245 & 035.15 & 5.19 & 01.475 & 071.353 \\
\hline & $48 \mathrm{hr}$. & 3.192 & 0124.645 & 039.05 & 5.37 & 02.212 & 056.349 \\
\hline & $72 \mathrm{hr}$. & 3.534 & 0151.417 & 042.85 & 5.38 & 02.507 & 060.398 \\
\hline & $96 \mathrm{hr}$. & 4.720 & 0599.557 & 127.02 & 6.68 & 05.383 & 111.380 \\
\hline & 120hr. & 8.322 & 0916.650 & 110.15 & 5.35 & 06.785 & 135.100 \\
\hline & 144hr. & 3.358 & 0188.083 & 056.01 & 5.97 & 07.374 & 025.506 \\
\hline
\end{tabular}

initial $\mathrm{pH}=6$, at $30 \circ \mathrm{C}$ 
Selim,A. E. I. et al.

Table 3b: Effect of glucose syrup, soy bean flour extract (SFE) and maize flour extract (MFE) on carotenoids production by Rhodotorula glutinis NRRL Y-842

\begin{tabular}{|c|c|c|c|c|c|c|c|}
\hline $\begin{array}{l}\text { Carbon } \\
\text { source }\end{array}$ & Time & \begin{tabular}{|c|} 
Cell dry \\
weight \\
(gm/litre)
\end{tabular} & $\begin{array}{c}\text { Carotenoids } \\
(\mu \mathrm{g} / \text { litre })\end{array}$ & 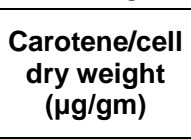 & $\begin{array}{c}\text { Final } \\
\mathrm{pH}\end{array}$ & $\begin{array}{c}\text { Consumed } \\
\text { sugars } \\
\text { (gm/liter) }\end{array}$ & $\begin{array}{c}\text { Carotene / } \\
\text { Consumed } \\
\text { Sugar } \\
(\mu \mathrm{g} / \mathrm{gm})\end{array}$ \\
\hline \multirow{6}{*}{$\begin{array}{l}\text { Glucose } \\
\text { syrup }\end{array}$} & 24hr. & 01.224 & 0087.30 & 071.32 & 5.85 & 01.178 & 074.109 \\
\hline & $48 \mathrm{hr}$. & 04.746 & 0419.04 & 088.29 & 6.83 & 02.462 & 170.203 \\
\hline & $72 \mathrm{hr}$. & 07.044 & 0465.60 & 066.10 & 6.05 & 10.011 & 046.509 \\
\hline & $96 \mathrm{hr}$. & 11.248 & 1831.36 & 162.85 & 5.71 & 14.133 & 129.580 \\
\hline & $120 \mathrm{hr}$. & 10.310 & 1431.72 & 138.87 & 5.68 & 14.186 & 100.925 \\
\hline & 144hr. & 09.060 & 1117.44 & 123.34 & 5.55 & 17.720 & 063.061 \\
\hline \multirow{6}{*}{ SFE } & 24hr. & 3.3067 & 247.67 & 74.90 & 6.05 & 01.387 & 178.565 \\
\hline & $48 \mathrm{hr}$. & 6.1000 & 426.80 & 69.97 & 6.96 & 11.891 & 035.893 \\
\hline & $72 \mathrm{hr}$. & 7.3067 & 267.07 & 36.55 & 7.43 & 17.941 & 014.886 \\
\hline & 96hr. & 8.9667 & 254.14 & 28.34 & 7.99 & 20.883 & 012.170 \\
\hline & $120 \mathrm{hr}$. & 9.1800 & 232.80 & 25.36 & 9.53 & 21.555 & 010.800 \\
\hline & 144hr. & 7.7933 & 132.89 & 17.05 & 9.23 & 22.058 & 006.025 \\
\hline \multirow{6}{*}{ MFE } & 24hr. & 3.5000 & 224.39 & 64.11 & 6.07 & 00.379 & 592.058 \\
\hline & $48 \mathrm{hr}$. & 6.2533 & 340.79 & 54.50 & 6.12 & 03.227 & 105.606 \\
\hline & $72 \mathrm{hr}$. & 6.9400 & 069.19 & 09.97 & 5.98 & 07.278 & 009.507 \\
\hline & 96hr. & 7.1733 & 060.14 & 08.38 & 6.06 & 14.050 & 004.280 \\
\hline & $120 \mathrm{hr}$. & 7.3400 & 060.14 & 08.19 & 6.07 & 18.481 & 003.254 \\
\hline & 144hr. & 6.8067 & 053.67 & 07.88 & 6.07 & 19.177 & 002.799 \\
\hline
\end{tabular}

initial $\mathrm{pH}=6$, at $30 \circ \mathrm{C}$

Data show differences in dry cell concentration and carotenoids production which may be due to composition, sugar, amino acids, vitamins, trace elements types and contents of the growth media, especially when agro-industrial by-products were used. Similar results were obtainted by Aksu and Tuğba Eren (2007).

Data reveal that, high amounts of sugars were consumed by $R$. glutinis NRRL Y- 842with the used carbon sources except in case of lactose, treated and untreated vinasse, where the consumed sugars were very low.

The $\mathrm{pH}$ of fermentation media increased during the 6 days of fermentation from an initial $\mathrm{pH} 6$ to $9.23,8.44$ and 7.35 in soy flour extract, sucrose and maltose treatments, respectively. It decreased from 6 to 4.6 and 4.62 in untreated beet molasses and lactose treatments, respectively. Data reveal that maximum carotenoid production seemed to occur after the exponential phase. Results are in a good agreement with some previous reports (Buzzini and Martini 1999 and Frengova et al. 1994) and divergently from other (Shih and Hang 1996).

In general, data reveal that carotenoids production is affected by Rhodotorula strain and carbon source. Results are in accordance with those reported by Buzzini and Martini (1999), who found that carotenoid production was affected by Rhodotorula strain and carbon source.

Regarding to the effect of time course on carotenoid production by $R$. glutinis NRRL-Y-842, it could be noticed that it gradually increased during the fermentation period, tell reaching the maximum value after $72,96,120$ or 144 hr. according to the used carbon source. Gamal et al., (2007) reported that 
the maximum carotenoids production by Rhodotorula glutinis 32 obtained after $72 \mathrm{hrs}$. using bioreactor.

In conclusion, it could be stated that the maximum values of carotenoids produced by $R$. glutinis NRRL-Y-842 were obtained using glucose $(2328.29 \mu \mathrm{g} / \mathrm{L}$ at $96 \mathrm{hr}$.), fructose $(2166.01 \mu \mathrm{g} / \mathrm{L}$ at $96 \mathrm{hr}$.), glucose syrup (1831.36 $\mu \mathrm{g} / \mathrm{L}$ at $96 \mathrm{hr}$.), untreated sugarcane molasses $(1772.19 \mu \mathrm{g} / \mathrm{L}$ at $120 \mathrm{hr}$.), maltose $(1704.97 \mu \mathrm{g} / \mathrm{L}$ at $96 \mathrm{hr}$.) and untreated beet molasses (1571.40 $\mathrm{\mu g} / \mathrm{L}$ at $144 \mathrm{hr}$.$) .$

The capability of $R$. glutinis NRRL-Y-842 for growing on a variety of carbon sources and agro-industrial by-products is a remarkable advantage. Data indicated that $R$. glutinis will be one of the most promising yeast for the commercial production of carotenoids by the use of agricultural by-products as cheap carbon sources. So, carotenoid productivity by $R$. glutinis is also favorable and worthwhile.

\section{REFERENCES}

Aksu, Z. and Tuğba Eren, A. (2005). Carotenoids production by the yeast Rhodotorula mucilaginosa: Use of agricultural wastes as carbon source. Process Biochemistry, 40: 2985-2991.

Aksu, Z. and Tuğba Eren, A. (2007). Production of carotenoids by the isolated yeast of Rhodotorula glutinis, Biochem. Eng. J., 35:107-113.

Ali, Dina F.I. (2013).Studies on carotenoids production from some yeasts.M.Sc.Thesis, Microbiol. Dept., Fac. of Agric., Mansoura Univ., Mansoura, Egypt.

Bhosale, P. and Gadre, R.V. (2001a). Production of $\beta$-carotene by a Rhodotorula glutinis mutant in sea water medium. Bioresource Technology, 76: 53-55.

Bhosale, P. and Gadre, R.V. (2001b). $\beta$-Carotene production in sugarcane molasses by a Rhodotorula glutinis mutant. J. Ind. Microbiol. Biotechnol., 26:327-332.

Buzzini, P. and Martini, A., (1999). Production of carotenoids by strains of Rhodotorula glutinis cultured in raw materials of agro-industrial origin. Bioresour. Technol., 71: 41-44.

Catalina Voaides and R.Dima(2012). The effect of nitrogen source on carotenoids production by Rhodotorula $s p$. Romanian Biotechnological Letters, 17(5):7570-7576.

Ferrao, M. and Garg, S. (2011). Studies on effect of media components on growth and $\beta$-carotene production by Rhodotorula graminis RC04. J. Cell and Tiss- ue Res., 11(1): 2551-2556.

Frengova, G.I., Simova, E.D., Pavlov, K., Beshkova, D.M., Grigorova, D. (1994). Formation of carotenoids by Rhodotorula glutinis in whey ultrafiltrate. Biotechnol. Bioeng., 44: 888-894. 
Selim,A. E. l. et al.

Frengova, G.I. and Beshkova, D.M.(2009). Carotenoids from Rhodotorula and Pha- ffia: yeasts of biotechnological importance. J. Ind. Microbiol. Biotechnol., 36: 163 - 180.

Gamal,Rawia F.; Abd El.hady, Hemmat M.; Selim, Sh.M. and Hussein, A.F. (2007). Evaluation of carotenoids produced by Rhodotorula glutinis using bioreactor with different feeding techniques on albino rats. Annals Agric.Sci., Ain Shams Univ., Cairo, 52(1):61-73.

Gordon, H.T. and J.C. Pauerfeind, (1982). Carotenoids as food colorants. Crit. Rev. Food Sci. Nutr., 18:59-97

Johnson, E. and Schroeder, W., (1995). Advanced in biochemical engineering /biot-echnology, in Fiechter A. (ed), Microbial carotenoids, 53, Berlin, $119-178$.

Park, P.K.; Cho, D.H.; Kim, E.Y. and Chu, K.H. (2005). Optimization of caroten-oid production by Rhodotorula glutinis using statistical experimental design. World J. Microbiol. Biotechnol., 21: 429-434.

Peterson, W. J.; Evans, W. R.; Leecce, E.; Bell, T.A. and Etchells, J. L. (1958). Q- uantitative determination of the carotenoids in yeast of the genus Rhodotorula. J.Bacteriol., 75:586-591.

Shih,C.T.and Hang, Y.D., (1996). Production of carotenoids by Rhodotorula rubra from sauerkraut brine. Lebensm. Wiss. Technol., 29: 570-572.

Somogyi, M. (1952). Notes on sugar determination. J. Biol. Chem., 195:19.

$$
\begin{aligned}
& \text { تأثير بعض السكريات ومخلفات الصناعات الزراعية على إنتاج الكاروتينات من بعض }
\end{aligned}
$$

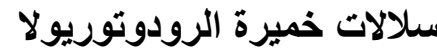

$$
\begin{aligned}
& \text { عبد الله العوضى إبراهيم سليم و محمد منصور الرواتو قاسم وسامية محمد مرسى بيومى ومحمود }
\end{aligned}
$$

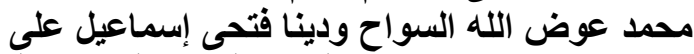

$$
\begin{aligned}
& \text { قسم الميكروبيولوجيا الزراعية ـ كلية ازراعة - جامعة المنصورة ـ المنصورة ـ مصر }
\end{aligned}
$$

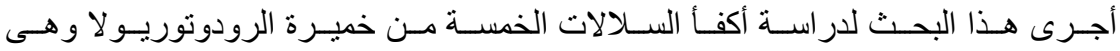

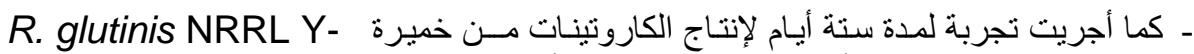
842 و استخدمت فيها أربعة سكريات وتحققت أعلى معدلات الإنتاج مع كل سكر كما يلى : 


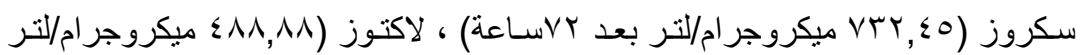

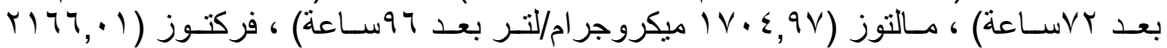
ميكروجر ام/لتر بعد 97 بـاعة ، مذاعة).

ـ كذلك أجريت تجربـة لإنتاج الكاروتينات من

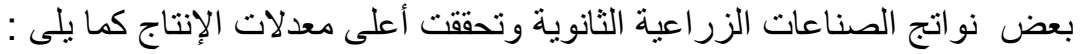

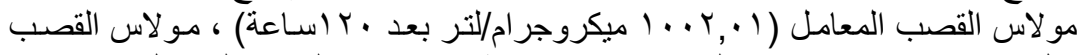

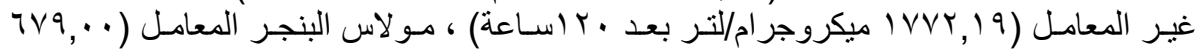

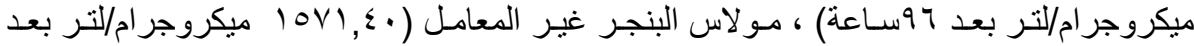

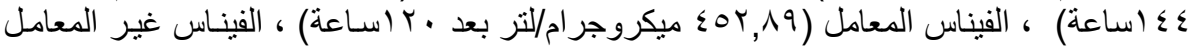

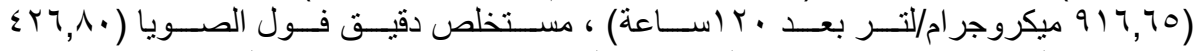

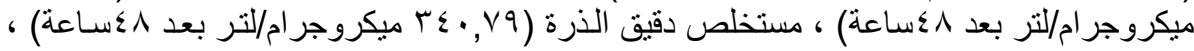

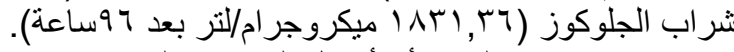

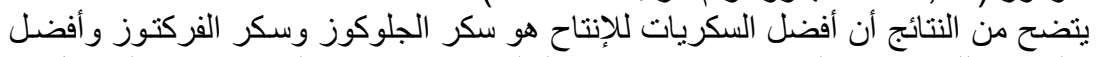

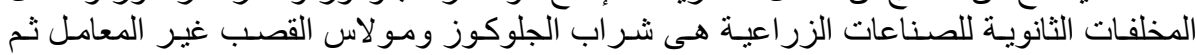

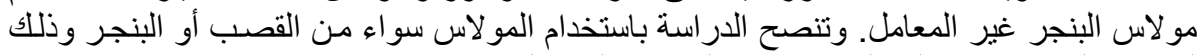

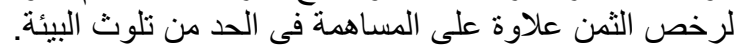

كلية الزراعة - جامعة المنصورة كلية الزراعة - جامعة دمياط
قام بتحكيم البحث

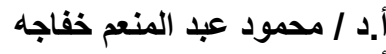

أ.د / حسين عبد الله الفضالى عمد خله 